\title{
A Survey on Load based Routing Techniques
}

\author{
Anurag Mehta \\ Department of Information Technology, \\ Medi-Caps Institute of Technology and Management, \\ Indore
}

\begin{abstract}
MANET is a collection of independent mobile nodes where any fixed infrastructure is not available for reliable and efficient communication.In this network all devices are works sender, receiver and router and data is delivered in multiple hops. This routing technique is backbone of the MANET network. Due to its ad hoc and dynamic nature, stable and reliable routing required. This paper provides a study about various enhanced routing techniques proposed and implemented previously, for finding stable and efficient routing. Additionally, based on previously defined problems, a new solution is proposed and described in this paper,and future extension of the proposed routing strategy is provided.
\end{abstract}

\section{Keywords}

MANET, performance issues, routing, future extension

\section{INTRODUCTION}

MANET (mobile ad hoc network) is defined by its own characteristics; it is self-organizing, mobile communication manner where topologies are dynamically created. Due to the ad hoc nature of the network infrastructure and mobility it is still an area of new research and development. Due to lack of infrastructure various issues arise in the network i.e. security, performance and simulation.

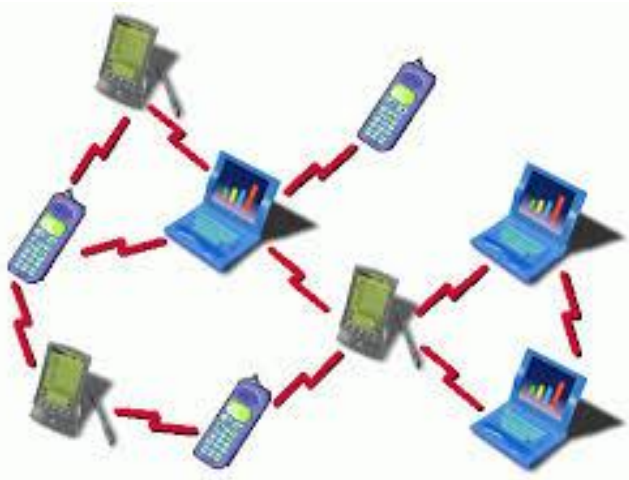

Fig 1.1

MANET simulates some characteristics by which the network id differentiated from the other kind of networks.

1. Each node act as both host and router. Therefore it is autonomous in behavior.

2. Multi-hop radio relaying- When a source node and target node for a message is out of the radio range, the MANETs is competent of multi-hop routing.

3. Distributed nature of operation of security, routing and host configuration. A centralized firewall is absent here.

4. The nodes can join or depart the network anytime, making the network topology active in nature.
5. Mobile nodes are illustrated with less memory, power and light weight features.

6. The reliability, efficiency, stability and capacity of wireless links are often inferior when evaluated with wired links. This shows the fluctuating link bandwidth of wireless links.

7. Mobile and impulsive behavior which demands least human intervention to configure the network.

8. All the nodes have similar features with similar responsibilities and abilities. Hence, it forms a completely symmetric environment.

9. High user density and large level of user mobility.

10. Nodal connectivity is intermittent.

A MANET environment has to overcome certain issues of restriction and inefficiency. It includes:

Zthe previously implemented some important contributions by various authors.

\section{BACKGROUND}

Before exploring about the contribution of different routing techniques and related work, some well-known routing techniques are discussed here.

An ad hoc routing protocol is a convention, or standard, that controls how nodes decide in which way to route packets between computing devices in a mobile ad hoc network. In ad hoc networks, nodes do not start out common with the topology of their networks; instead, they have to determine it. The basic idea is that a new node may announce its presence and should listen to announcements broadcast by its neighbors. Each node learns about nodes nearby and how to reach them, and may announce that it, too, can reach them.

Proactive (table-driven) routing: This type of protocols retains fresh lists of destinations and their routes by periodically allocating routing tables throughout the network. The main drawbacks of such algorithms are:

1. Respective amount of data for maintenance.

2. Slow reaction on restructuring and failures.

Reactive (on-demand) routing: This type of protocols discovers a route on order by flooding the network with Route Request packets. The main drawbacks of such algorithms are:

1. High latency time in route finding.

2. Extreme flooding can lead to network clogging

Flow-oriented routing: This type of protocols discovers a route on demand by following present flows. One option is to unicast successively when forwarding data while promoting a new link. The main drawbacks of such algorithms are 
1. Takes long time when investigates new routes without a previous knowledge.

2. May refer to existing traffic to compensate for omitted knowledge on routes.

Hybrid (both pro-active and reactive): This type of protocols combines the advantages of proactive and of reactive routing. The routing is primarily established with some proactively prospected routes and then provides the demand from additionally activated nodes through reactive flooding. The selection for one or the other method needs predetermination for typical cases. The main drawbacks of such algorithms are:

1. Advantage depends on the number of nodes activated.

2. Reacting to traffic demand depends on the gradient of traffic volume.

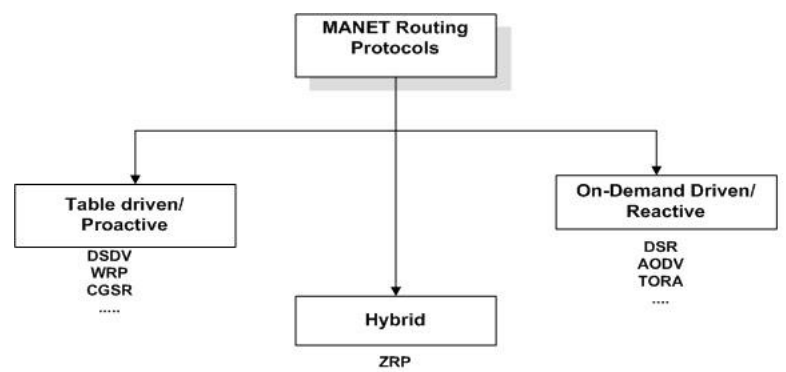

Fig 1.2

Hierarchical routing protocols: With this type of protocols the selection of proactive and of reactive routing depends on the hierarchic level where a node exists. The routing is primarily established with some proactively prospected routes and then orders the demand from additionally activated nodes through the reactive flooding on the lower levels. The choice for one or the other method requires proper attribution for the respective levels. The main drawbacks of such algorithms are:

1. Advantage depends on the depth of nesting and addressing scheme

2. Reacting to traffic demand depends on meshing parameters

Backpressure Routing: This type of routing does not precompute paths. It selects next-hops dynamically as a packet is in progress toward its target. These decisions are based on obstruction gradients of neighbor nodes. When this type of routing is used mutually with max-weight link scheduling, the algorithm is throughput-optimal. See further discussion here: Backpressure Routing.

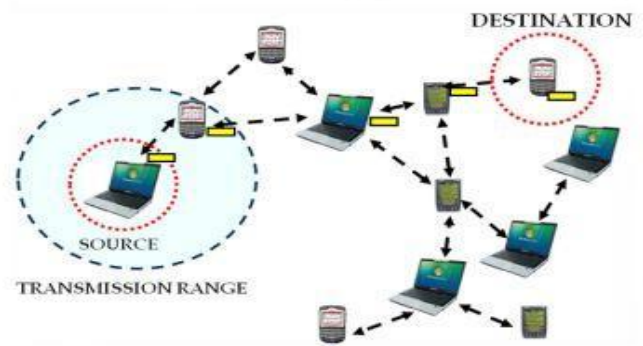

Fig 1.3

Host Specific Routing protocols: This type of protocols requires thorough administration to alter the routing to a definite network layout and a distinct flow strategy, the main drawbacks of such algorithms are:

1. Advantage depends on the quality of the administration addressing scheme.

2. The proper reaction to alters in topology demands reconsidering all parameters

Power-aware routing protocols: Energy needed to transmit a signal is approximately proportional to $d \alpha$, where the distance is $d$ and $\alpha>=2$ is the attenuation factor or the path loss exponent, which depends on the transmission medium. When $\alpha=2$ (which is the optimal case), transmitting a signal half the distance needs one fourth of the energy and if there is a node in the middle willing to spend another fourth of its energy in the second half, data would be transferred for half of the energy than through a direct transmission - a fact that follows directly from the inverse square law of physics. The main drawbacks of such algorithms are:

1. This method stimulates a delay for each transmission.

2. No relevance for energy network powered transmission operated via sufficient repeater infrastructure.

During study various kinds of routing strategies are available for end to end data delivery. In this paper our main domain of interest is working with energy and traffic based scheme. Some important contribution and efforts are also analyzed for finding a better approach for load aware routing techniques.

\section{LITERATURE STUDY}

In this section of the paper we describe previously made efforts and contributions over load aware routing technique is provided for previous work analysis and our literature collection.

[Yashar 2004] provides an important study about load balancing in ad hoc routing, according to author Multi-path routing has been studied systematically in the context of wired networks. It has been shown that using various paths to route messages between any source-destination pair of nodes (instead of using a single path) balances the load more consistently throughout the network. The universal belief is that the similar is true for ad hoc networks, i.e., multi-path routing balances the load significantly better than single-path routing.In this paper, we show that this is not essentially the case. Author introduces a new model for calculating the load balance under multi-path routing, when the paths chosen are the first $\mathrm{K}$ shortest paths (for a pre-specified $\mathrm{K}$ ). Using this model, they show that unless we use a very large number of paths (which is very costly and therefore infeasible) the load allocation is almost the similar as single shortest path routing. This is in dissimilar to the earlier existing results which imagine that multi-path routing spread the load uniformly.[1]

Mobile ad hoc networks (MANETs) are very capable wireless technology and they provide wide range of possibilities for the future in terms of applications and coverage. Due to the difficult nature of MANETS, their improvement processes face numerous challenges such as routing. Reactive routing protocols are favored and famous in MANETs because they are more scalable and create fewer overhead on the network. But, these protocols undergo from the broadcast storm trouble due to the flooding strategy that is used in the route discovery process which causes redundancy, contention and collision problems. In order to reduce the effects of the broadcast problem [Yaser 2011] provide, a Mobility and Load aware Routing scheme (MLR) is projected in this paper. MLR controls the flooding process by restricting there broadcast 
messages on the slow speed and low loaded nodes. Each node chooses whether to forward or drop the received request message based on numerous factors (such as speed and routing load) using Markovian Decision Process tool. Simulation results demonstrate that MLR scheme outperforms the original AODV protocol in terms of normalized routing load and average end-to-end delay. [2]

Another contribution based on load aware routing for wireless mesh network is given by [Lan 2011] This paper proposes a load and interference-aware routing metric for wireless mesh networks, named Channel Utilization and Contention Window Based (C2WB) metric. Our metric allocates weights to individual links which are proportional to the links service times projected from both channel utilization and the average contention window of the CSMA/CA mechanism. The path metric, joint from individual link weights, accounts for both load and interference of the links on the path. Thus the C2WB metric helps the routing protocol to balance the traffic and to advance the network capacity by avoiding routing the traffic through crowded areas. [3]

Mobile ad hoc networks are groups of mobile nodes that can dynamically form temporary networks without the require for pre-existing network infrastructure or centralized administration. These nodes can be randomly located and can travel freely at any given time. Hence, the network topology can change quickly and unpredictably. Because wireless link abilities are usually limited this study is given by [Chai 2009], congestion is possible inMANETs. Hence, balancing the load in a MANET is significant since nodes with high loads will reduce their batteries rapidly, thereby increasing the probability of disconnecting orpartitioning the network. This article discusses the various load metrics and explains the principles behind numerous existing load balanced ad hoc routing protocols. Finally, a qualitative evaluation of the various load metrics and load balanced routing protocols is presented. [4]

An important work with energy aware routing is placed by [Aruna 2010] in this work, author analyzes the design of green routing algorithms and evaluates the achievable energy savings that such mechanisms could allow in several realistic network scenarios. They formulate the problem as a minimum energy routing optimization, which we numerically solve considering a core-network scenario, which can be seen as a worst-case for energy saving performance (as nodes cannot be switched off). To gather full relief results, author analyzes the energy savings in various conditions (i.e., network topology and traffic matrix) and under different technology assumptions (i.e., the energy profile of the network devices).These results give us insight into the potential benefits of different "green" technologies and their interactions. In particular, they show that depending on the topology and traffic matrices, the optimal energy savings can be modest, partly limiting the interest for green routing approaches for some scenarios. At the same time, author also shows that the common belief that there is a tradeoff between green network optimization and performance does not necessarily holds: in the considered environment, green routing has no effect on the main network performances such as maximum link utilization. [5]

A research paper is given by [Wei 2011], this paper addresses energy conservation, a fundamental issue of paramount importance in heterogeneous mobile ad hoc networks (MANETs) consisting of powerful nodes (i.e.,P-nodes) as well as normal nodes (i.e., B-nodes). By utilizing the inherent device heterogeneity, we propose a cross-layer designed
Device-Energy-Load Aware Relaying framework, named DELAR, to achieve energy conservation from multiple facets, including power-aware routing, transmission scheduling and power control. In particular, we design a novel power-aware routing protocol that nicely incorporates device heterogeneity, nodal residual energy information and nodal load status to save energy. In addition, we develop a hybrid transmission scheduling scheme, which is a combination of reservationbased and contention-based medium access control schemes, to coordinate the transmissions. Moreover, the novel

notion of "mini-routing"is established into the data link layer and an Asymmetric MAC(A-MAC) scheme is proposed to maintain the MAC-layer acknowledgements over unidirectional links caused by asymmetric transmission power levels between dominant nodes and normal nodes. Additionally, we present a multi-packet transmission scheme to advance the end-to-end delay performance. Extensive simulations show that DELAR can indeed achieve energy saving while striking a good balance between energy efficiency and on the network performance metrics. [6]

In this paper,[Xu Li 2012]propose to apply the concept of cost to progress ratio (CPR) in geographic routing for load balancing. The resultant routing protocol is named CPRrouting. It is alocalized parameter-less approach optimizing both geographic progress and nodal load during hop selection. Through extensive simulation, author compares it with an existing parameter-based localized solution $\alpha$-routing. Given simulation results indicate that CPR-routing outperforms $\alpha$ routing in both load balancing and packet delivery ratio. [7]

This section provides the overview of the previously proposed routing that are affected by the network conditions such as energy and routing load. In the next section provides the summary of the previously defined techniques.

\section{LITERATURE EXTRACTION}

There are various efforts and techniques are available for improving the routing technique. Most of them are working with different parameters for finding the delivery; these methods improve the performance of the network considerably.

The most suitable and efficient way during the literature collection is found by [ $\mathrm{Xu} \mathrm{Li} \mathrm{2012].} \mathrm{In} \mathrm{this} \mathrm{technique} \mathrm{the}$ author consider that the energy is a best parameter for estimating the load, because of that is not recoverable once it is consumed. In this article author uses the energy consumption and a routing technique for improving the routing technique. And in this context they found that the performance of network is considerably efficient. But they can more improve the technique of routing, by considering some more facts about the routing and routing strategy. The next section provides the general idea of the proposed improvement over the existing routing technique.

\section{CONCLUSION AND FUTURE WORK}

In this work we are making efforts for design a new routing technique where the load parameters are decides the route from source to destination. Therefore the proposed technique includes the following key factors based on load routing technique, the proposed technique is also includes the mobility in nodes and also promises to provides the efficient routing during high traffic load. Some key features of the routing technique are given as:

Load is calculated using the energy consumption of the nodes. That is assumed that each node contains some initial energy 
and for sending data, receiving data and routing of data packets an amount of energy consumed. now there are two main factors are involved first if energy is below than a limit, that means node is not able to route more packets therefore required to eliminated the node form routing. Secondly if node is consumes energy much frequently that means the node is already in overload therefore that is also not able to handle more data with this route.

Therefore an efficient routing which is based on load awareness is required to implement using DSR routing algorithm, using NS2 simulation environment, the simulation of the proposed routing technique and the performance of that is provided in future work.

\section{REFERENCES}

[1] Load Balancing in Ad Hoc Networks:Single-path Routing vs. Multi-path Routing,YasharGanjali, AbtinKeshavarzian,0-7803-8356-7/04/\$20.00 $\quad$ (C) 2004 IEEE

[2] Mobility and Load aware Routing protocolfor ad hoc networks, YaserKhamayseh,

GhadeerObiedat, MunnerBani Yassin, 2011 King Saud University. Production and hosting by Elsevier
[3] AninterferenceandloadawareroutingmetricforWirelessMe shNetworks,LanTienNguyen,RazvanBeuran,YoichiShino da,Copyright (C) 2011 Inderscience Enterprises Ltd.

[4] Load Balanced Routing Protocols for Ad Hoc Mobile Wireless Networks,Chai KeongToh, University of Hong KongAnh-Ngoc Le and You-Ze Cho,01636804/09/\$25.00 @ 2009 IEEE

[5] Energy-Aware Routing: a Reality Check,ArunaPremBianzino, Claude Chaudet, Federico Larroca, Dario Rossi, Jean-Louis Rougier,978-1-4244$8864-3 / 10 / \$ 26.00$ @2010 IEEE

[6] DELAR: A Device-Energy-Load AwareRelaying Framework for Heterogeneous MobileAd Hoc Networks, Wei Liu, Chi Zhang, Guoliang Yao, andYuguang Fang,0733-8716/11/\$25.00c 2011 IEEE

[7] Localized Load Balancing for Geographic Routingin Wireless Ad Hoc Networks,Xu Li, Nathalie Mitton, Amiya Nayak, and Ivan Stojmenovic, Wireless Networks Symposium -( IEEE ICC-WN 2012) (2012) 